\title{
Some Properties of Soft Connected Spaces and Soft Locally Connected Spaces
}

\author{
${ }^{1}$ Assist. Prof. Dr. Munir Abdul Khalik Al-Khafaj, ${ }^{2}$ Majd Hamid Mahmood \\ Email:mnraziz@yahoo.com ${ }^{1}$,Email:mgmg227@yahoo.com ${ }^{2}$ \\ ${ }^{1,2}$ (Department of Mathematics, College of Education, Al-Mustansiriya University , Baghdad, Iraq)
}

\begin{abstract}
In this paper we will introduced soft disjoint sets, soft separated sets ,soft connected space, soft disconnected space, and study the relation between soft disjoint sets and soft separated sets, define soft hereditary property and study soft hereditary property on soft connected spaces, study the product of two soft disconnected spaces and the product of soft connected with soft disconnected .
\end{abstract}

\section{Introduction}

Soft sets was introduced by the Russian Demetry Molodtsove 1999 [2] as ageneral mathematical tool for dealing with uncertain objects, operations on soft set was introduced by P.K. Maji , R . Biswas and A. R. Roy 2003[9], Sabir and Nas 2011[8] introduce and study the concept of soft topological spaces over soft set and some related concepts, In 2011 [1] Aygunogla, Aygun introduced the soft product topology, E. Peygh and B. Samadi , A.Tayebi 2013[5] introduce soft locally connected of a soft point and soft connected spaces depending on soft disjoint non-null soft open sets .

Let $X$ be an initial universe and $\mathrm{E}$ be a set of parameters, $\mathrm{P}(\mathrm{X})$ denote the power set of $\mathrm{X}$, A pair $(\mathrm{F}, \mathrm{E})$ is called a soft set over $X$, where $F$ is a mapping given by $F: E \rightarrow P(X)$ For two soft sets $(F, A)$ and $(G, B)$ over common universe $X$, we say that $(F, E)$ is a soft subset $(G, E)$ if $A \subseteq B$ and $F(e) \subseteq G(e)$, for all e $\in A$, null soft set denoted by $\widetilde{\Phi}$ where if for each e $\in \mathrm{E}, \mathrm{F}(\mathrm{e})=\Phi$, absolute soft set denoted by $\widetilde{\mathrm{X}}$, if for each e $\in \mathrm{E}$, $F(e)=X$, union of two soft sets of $(F, A)$ and $(G, B)$ over the common universe $X$ is the soft set $(H, C)$, where

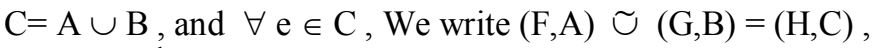

$$
H(e)= \begin{cases}F(e) & \text { if } e \in A-B \\ G(e) & \text { if } e \in B-A \\ F(e) \cup G(e) & \text { if } e \in A \cap B\end{cases}
$$

the intersection of two soft sets $(\mathrm{F}, \mathrm{A})$ and $(\mathrm{G}, \mathrm{B})$ over a common universe $\mathrm{X}$ is the soft set $(\mathrm{H}, \mathrm{C})$, where

$\mathrm{C}=\mathrm{A} \cap \mathrm{B}$, and, $\mathrm{H}(\mathrm{e})=\mathrm{F}(\mathrm{e}) \cap \mathrm{G}(\mathrm{e})$, We write $(\mathrm{F}, \mathrm{A}) \widetilde{\cap}(\mathrm{G}, \mathrm{B})=(\mathrm{H}, \mathrm{C}) \forall \mathrm{e} \in \mathrm{C}$,

$\mathrm{SS}(\mathrm{X})_{\mathrm{E}}$ denote to the set of all soft sets $(\mathrm{F}, \mathrm{E})$ over a universe $\mathrm{X}$ in which all parameter $\mathrm{E}$ are the same.

Definition1.1.[8] Let $\widetilde{T}$ be the collection of soft sets over $\mathrm{X}$ then $\widetilde{T}$ is soft topology on $\mathrm{X}$ if :

1- $\widetilde{\Phi}, \widetilde{X}$ belong to $\widetilde{T}$.

2- The union of any number of soft sets in $\widetilde{T}$ belongs to $\widetilde{T}$.

3- The intersection of any two soft sets in $\widetilde{T}$ belongs to $\widetilde{T}$.

The triple $(X, \widetilde{T}, E)$ is called a soft topological space over $X$ denoted by (S.T.S.)

Definition1.2. [8] Let $(X, \widetilde{T}, E)$ be a S.T.S over $X$ then the members of $\widetilde{T}$ are said to be soft open sets in $X$.

Definition1.3. [8] Let $(X, \widetilde{T}, E)$ be a S.T.S over $X$, a soft set $(F, E)$ over $X$ is said to be a soft closed set in $X$, if its complement $(\mathrm{F}, \mathrm{E})^{\prime}$ belongs to $\widetilde{\mathrm{T}}$.

Definition1.4.[3] Let $(F, E) \widetilde{\in} S S(X)_{E}$ we say that $\widetilde{\boldsymbol{x}}=(\mathrm{e},\{\mathrm{h}\})$ is a nonempty soft element of $(\mathrm{F}, \mathrm{E})$ if e $\in \mathrm{E}$ and

$h \in F(e)$. The pair $(e, \Phi)$ is called an empty soft element of $(F, E)$.Nonempty soft elements of $(F, E)$ and empty soft elements of $(F, E)$ are called the soft elements of $(F, E)$.

Remark 1.5.[3] If $\widetilde{\boldsymbol{x}}$ is a soft element of (F,E) will be denoted by $\widetilde{\boldsymbol{x}} \widetilde{\in}(\mathrm{F}, \mathrm{E})$.

Example 1.6. Let $X=\left\{h_{1}, h_{2}, h_{3}\right\}, E=\left\{e_{1}, e_{2}\right\}$. Take a soft set $(F, E) \widetilde{\in} S S(X)_{E}$ of the form

$(F, E)=\left\{\left(e_{1},\left\{h_{1}\right\}\right),\left\{\left(e_{2},\left\{h_{1}, h_{2}\right\}\right)\right\}\right.$. Then all the soft elements of $(F, E)$ are the following: $\left(\mathrm{e}_{1}, \Phi\right),\left(\mathrm{e}_{1},\left\{\mathrm{~h}_{1}\right\}\right)$,

$\left(\mathrm{e}_{2}, \Phi\right),\left(\mathrm{e}_{2},\left\{\mathrm{~h}_{1}\right\}\right),\left(\mathrm{e}_{2},\left\{\mathrm{~h}_{2}\right\}\right)$.

Definition 1.7.[4] Let (F,E) be a soft set over X, $Y$ be a non empty subset of $X$ then the soft subset (F,E) over $Y$ denoted by $\left({ }^{\mathrm{Y}} \mathrm{F}, \mathrm{E}\right)$ is defined as follows

${ }^{\mathrm{Y}} \mathrm{F}(\mathrm{e})=\mathrm{Y} \cap \mathrm{F}(\mathrm{e})$ for each $\mathrm{e} \in \mathrm{E}$ (in other word $\left.\left({ }^{\mathrm{Y}} \mathrm{F}, \mathrm{E}\right)=\widetilde{\mathrm{Y}} \widetilde{\cap}(\mathrm{F}, \mathrm{E})\right)$.

Definition1.8.[4] Let (X, $\widetilde{T}, E)$ be soft topological space over $X$ and $Y$ be a non empty subset of $X$, then $\widetilde{\mathrm{T}}_{\mathrm{Y}}=\left\{\left({ }^{\mathrm{Y}} \mathrm{F}, \mathrm{E}\right) \mid(\mathrm{F}, \mathrm{E}) \in \widetilde{\mathrm{T}}\right\}$

is said to be the soft relative topology on $\mathrm{Y}$ and $\left(\mathrm{Y}, \widetilde{\mathrm{T}}_{\mathrm{Y}}, \mathrm{E}\right)$ is called soft subspace topology of $(\mathrm{X}, \widetilde{T}, \mathrm{E})$. 


\section{Soft Disjoint And Soft Separated}

In this section we will introduced soft disjoint sets and study the relation between soft disjoint sets and soft separated sets define soft disconnected spaces depending on soft separated sets .

Definition 2.1. Two soft subsets (F,E), (G,E) are said to be soft disjoint if

$(\mathrm{F}, \mathrm{E}) \widetilde{\cap}(\mathrm{G}, \mathrm{E})=\widetilde{\Phi}$.

Definition 2.2. Let (X, $\widetilde{T}, \mathrm{E})$ be a S.T.S., two non-null soft subsets $(\mathrm{F}, \mathrm{E}),(\mathrm{G}, \mathrm{E})$ of $(\mathrm{X}, \widetilde{T}, \mathrm{E})$ are said to be soft separated of $\widetilde{\mathrm{X}}$ iff $(\mathrm{F}, \mathrm{E}) \widetilde{\cap} \mathrm{cl}(\mathrm{G}, \mathrm{E})=\widetilde{\Phi}$ and

$\operatorname{cl}(\mathrm{F}, \mathrm{E}) \widetilde{\cap}(\mathrm{G}, \mathrm{E})=\widetilde{\Phi},(\mathrm{F}, \mathrm{E}) \widetilde{\sim}(\mathrm{G}, \mathrm{E})=\widetilde{\mathrm{X}},(\mathrm{F}, \mathrm{E}) \widetilde{\cap}(\mathrm{G}, \mathrm{E})=\widetilde{\Phi}$

Example 2.3. $\mathrm{X}=\{\mathrm{a}, \mathrm{b}, \mathrm{c}\}, \mathrm{E}=\left\{\mathrm{e}_{1}, \mathrm{e}_{2}\right\}, \mathrm{T}=\{\widetilde{\Phi}, \widetilde{\mathrm{X}},(\mathrm{F}, \mathrm{E}),(\mathrm{G}, \mathrm{E})\}$ where

$\mathrm{F}\left(\mathrm{e}_{1}\right)=\{\mathrm{a}\}, \mathrm{F}\left(\mathrm{e}_{2}\right)=\{\mathrm{b}, \mathrm{c}\}, \mathrm{G}\left(\mathrm{e}_{1}\right)=\{\mathrm{b}, \mathrm{c}\}, \mathrm{G}\left(\mathrm{e}_{2}\right)=\{\mathrm{a}\}$

$(F, E),(G, E)$ are Soft separated of $\widetilde{X}$ and also soft disjoint of $\widetilde{X}$

Remark 2.4. In a S.T.S. any two soft separated sets are soft disjoint sets but two disjoint soft sets are not necessary soft separated sets.

Example 2.5. Let $\mathrm{X}=\{\mathrm{a}, \mathrm{b}, \mathrm{c}, \mathrm{d}\}, \mathrm{E}=\left\{\mathrm{e}_{1}, \mathrm{e}_{2}, \mathrm{e}_{3}, \mathrm{e}_{4}, \mathrm{e}_{5}\right\}, \mathrm{F}_{1}\left(\mathrm{e}_{1}\right)=\{\mathrm{a}\}, \mathrm{F}_{2}\left(\mathrm{e}_{1}\right)=\{\mathrm{b}, \mathrm{c}, \mathrm{d}\}$

$\mathrm{T}=\left\{\widetilde{\Phi}, \widetilde{\mathrm{X}},\left(\mathrm{F}_{1}, \mathrm{E}\right),\left(\mathrm{F}_{2}, \mathrm{E}\right)\right\}$ is soft topology, $\left(\mathrm{F}_{1}, \mathrm{E}\right),\left(\mathrm{F}_{2}, \mathrm{E}\right)$ are two disjoint soft sets but not soft separated sets .

Theorem 2.6. Two soft close (soft open) subsets (F,E), (G,E) of a S.T.S. (X, T,E) are soft separated if and only if they are soft disjoint.

Proof $(\Rightarrow)$ it's clear that any two separated sets are disjoint.

$(\Leftarrow)$ Let $(\mathrm{F}, \mathrm{E}),(\mathrm{G}, \mathrm{E})$ are both soft disjoint and soft close sets then

So that $\mathrm{cl}(\mathrm{F}, \mathrm{E}) \widetilde{\cap}(\mathrm{G}, \mathrm{E})=\widetilde{\Phi}$ and $\mathrm{cl}(\mathrm{G}, \mathrm{E}) \widetilde{\cap}(\mathrm{F}, \mathrm{E})=\widetilde{\Phi}$

Showing that $(F, E),(G, E)$ are soft separated.

If (F,E), (G,E) are both soft disjoint and soft open sets then (F,E)', (G,E)' are both soft close

Then $(\mathrm{F}, \mathrm{E}) \widetilde{\subseteq}(\mathrm{G}, \mathrm{E})^{\prime}, \quad(\mathrm{G}, \mathrm{E}) \widetilde{\subseteq}(\mathrm{F}, \mathrm{E})^{\prime}, \operatorname{cl}(\mathrm{F}, \mathrm{E}) \widetilde{\subseteq} \mathrm{cl}(\mathrm{G}, \mathrm{E})^{\prime}=(\mathrm{G}, \mathrm{E})^{\prime}$ and $\operatorname{cl}(\mathrm{G}, \mathrm{E}) \widetilde{\subseteq} \mathrm{cl}(\mathrm{F}, \mathrm{E})^{\prime}=(\mathrm{F}, \mathrm{E})^{\prime}$

$\Rightarrow \operatorname{cl}(\mathrm{F}, \mathrm{E}) \widetilde{\cap}(\mathrm{G}, \mathrm{E})=\widetilde{\Phi}$ and $\mathrm{cl}(\mathrm{G}, \mathrm{E}) \widetilde{\cap}(\mathrm{F}, \mathrm{E})=\widetilde{\Phi}$ therefore $(\mathrm{F}, \mathrm{E})$ and $(\mathrm{G}, \mathrm{E})$ are soft separated.

Theorem 2.7. If $\left(X, \widetilde{T}_{1}, E\right)$ is soft separated and $\widetilde{T}_{1} \simeq \widetilde{T}_{2}$ then $\left(X, \widetilde{T}_{2}, E\right)$ is soft separated

Proof suppose $\left(X, \widetilde{T}_{1}, E\right)$ is soft separated,$\widetilde{T}_{2}$ is soft topology on $X$ finer then $\widetilde{T}_{1}$, T.P. that $\left(X, \widetilde{T}_{2}, E\right)$ is soft separated since $\left(X, \widetilde{T}_{1}, E\right)$ soft separated implies that there exist non-null soft subset $(\mathrm{F}, \mathrm{A})$ of $\left(\mathrm{X}, \widetilde{\mathrm{T}}_{1}, \mathrm{E}\right)$ which both $\widetilde{\mathrm{T}}_{1}$ - soft open $\widetilde{\mathrm{T}}_{1}$ - soft close set.

Since $\widetilde{\mathrm{T}}_{1} \widetilde{\subseteq}_{\overparen{T}} \Rightarrow(\mathrm{F}, \mathrm{E})$ is $\widetilde{\mathrm{T}}_{2}$ - soft open set, $(\mathrm{F}, \mathrm{E}) \widetilde{\mathrm{T}}_{1}$ - soft close set

Then (F,E)' is $\widetilde{T}_{1}$ - soft pen set $\Rightarrow(\mathrm{F}, \mathrm{E})^{\prime}$ is $\widetilde{\mathrm{T}}_{2}$-soft open set $\Rightarrow(\mathrm{F}, \mathrm{E})$ is $\widetilde{\mathrm{T}}_{2}$ - soft close set .Thus (F,E) non-null proper soft subset of $X$ which is both $\widetilde{T}_{2}$ - soft open $\widetilde{T}_{2}$ - soft close in $\left(X, \widetilde{T}_{2}, E\right)$ then it is soft separated.

\section{Soft Disconnected Space And Soft Connected Space}

Definition 3.1. A S.T.S. (X, $\widetilde{T}, E)$ is soft disconnected iff there exist two non-null soft open subsets $(F, E)$ and (G,E) of $(X, \widetilde{T}, E)$ s.t.

1. $(\mathrm{F}, \mathrm{E}) \widetilde{\sim}(\mathrm{G}, \mathrm{E})=\widetilde{\mathrm{X}}, 2 \cdot \operatorname{cl}(\mathrm{F}, \mathrm{E}) \widetilde{\cap}(\mathrm{G}, \mathrm{E})=\widetilde{\Phi}$ and $(\mathrm{F}, \mathrm{E}) \widetilde{\cap} \mathrm{cl}(\mathrm{G}, \mathrm{E})=\widetilde{\Phi}$

(i.e. $\widetilde{X}$ is the union of two non-null soft separated sets).

Example 3.2. Let $X \neq \Phi$ be a set of laptops, $E=$ \{good quality, high quality

Where $F_{1}($ good quality $)=F_{2}($ high quality $)=F_{3}($ good quality $)=F_{3}$ (high quality $)=\Phi, F_{1}$ (high quality $)=F_{2}($ good quality $)=F_{4}($ good quality $)=F_{4}($ high quality $)=X$

Then $\mathrm{T}=\left\{\left(\mathrm{F}_{1}, \mathrm{E}\right),\left(\mathrm{F}_{2}, \mathrm{E}\right),\left(\mathrm{F}_{3}, \mathrm{E}\right),\left(\mathrm{F}_{4}, \mathrm{E}\right)\right\}$ is soft topology

$\left(F_{1}, E\right),\left(F_{2}, E\right)$ form a soft separation of $\widetilde{X}$ therefore $(X, \widetilde{T}, E)$ is soft disconnected notice that the space $(X, \widetilde{T}, E)$ even when $\mathrm{X}$ is only one laptop can be soft disconnected.

Definition 3.3. A S.T.S. (X, $\widetilde{T}, E)$ is said to be soft connected space iff it is not disconnected .

Examples3.4.

1- let $\mathrm{X}=\left\{\mathrm{h}_{1}, \mathrm{~h}_{2}, \mathrm{~h}_{3}\right\}$ be the set of three houses, $\mathrm{E}=\left\{\mathrm{e}_{1}=\right.$ expensive, $\mathrm{e}_{2}=$ repaired $\}$ be the set of parameters ,then the soft sets which is defined as follows $F\left(e_{1}\right)=\Phi, F\left(e_{2}\right)=\Phi, G\left(e_{1}\right)=X, G\left(e_{2}\right)=X$ generate a soft connected soft topological space .

2- The soft singleton set is soft connected and null soft set is soft connected .

Notice 3.5 .

(i) The soft subset of soft connected space is not necessary soft connected as seen in Example (4.9.) the soft set $\left\{\left\{\mathrm{h}_{1}, \mathrm{~h}_{2}\right\}, \Phi, \Phi\right\}$ is disconnected set in a soft connected topological space $\left(\mathrm{Y}, \widetilde{\mathrm{T}}_{\mathrm{Y}}, \mathrm{E}\right)$.

(ii) The soft subset of soft disconnected space is not necessary soft disconnected as seen in Example (4.10.) the soft set $\left\{\left\{\mathrm{h}_{1}\right\},\left\{\mathrm{h}_{1}\right\},\left\{\mathrm{h}_{1}\right\}\right\}$ is soft connected set in a soft disconnected topological space $\left(\mathrm{Y}, \widetilde{\mathrm{T}}_{\mathrm{Y}}, \mathrm{E}\right)$.

Definition3.6.a soft subspace $\left(\mathrm{Y}, \widetilde{\mathrm{T}}_{\mathrm{Y}}, \mathrm{E}\right)$ of a S.T.S. $(\mathrm{X}, \widetilde{\mathrm{T}}, \mathrm{E})$ is soft disconnected if and only $\widetilde{\mathrm{Y}}$ is the a union of two non-null disjoint soft clopen sets in $\widetilde{Y}$. 
Theorem 3.7. Two disjoint soft sets $(F, E),(G, E)$ are soft separated in a S.T.S.

$(\mathrm{X}, \widetilde{T}, \mathrm{E})$ iff they are both soft clopen in the soft subspace $(\mathrm{F}, \mathrm{E}) \widetilde{\sim}(\mathrm{G}, \mathrm{E})=\widetilde{\mathrm{Y}}$

Proof $(\Rightarrow)$ Let $(F, E),(G, E)$ the soft disjoint set be soft separated in $(X, \widetilde{T}, E)$ so that

$(\mathrm{F}, \mathrm{E}) \widetilde{\cap} \mathrm{cl}_{\mathrm{x}}(\mathrm{G}, \mathrm{E})=\widetilde{\Phi}$ and $\mathrm{cl}_{\mathrm{x}}(\mathrm{F}, \mathrm{E}) \widetilde{\cap}(\mathrm{G}, \mathrm{E})=\widetilde{\Phi}$

Let $\widetilde{Y}=(\mathrm{F}, \mathrm{E}) \widetilde{\sim}(\mathrm{G}, \mathrm{E})$ then

$\mathrm{cl}_{\mathrm{Y}}(\mathrm{F}, \mathrm{E})=\mathrm{cl}_{\mathrm{x}}(\mathrm{F}, \mathrm{E}) \widetilde{\cap} \widetilde{\mathrm{Y}}=\mathrm{cl}_{\mathrm{x}}(\mathrm{F}, \mathrm{E}) \widetilde{\cap}[(\mathrm{F}, \mathrm{E}) \widetilde{\sim}(\mathrm{G}, \mathrm{E})]$

$\left.=\left[\mathrm{cl}_{\mathrm{x}}(\mathrm{F}, \mathrm{E}) \widetilde{\cap}(\mathrm{F}, \mathrm{E})\right] \widetilde{\mathrm{cl}_{\mathrm{x}}}(\mathrm{F}, \mathrm{E}) \widetilde{\cap}(\mathrm{G}, \mathrm{E})\right]=(\mathrm{F}, \mathrm{E}) \widetilde{\circlearrowleft} \widetilde{\Phi}=(\mathrm{F}, \mathrm{E})$

Hence $(F, E)$ is soft closed subspace of $\widetilde{Y}$

Similarly $(\mathrm{G}, \mathrm{E})$ is soft closed subspace in $\widetilde{\mathrm{Y}}$, and since $(\mathrm{F}, \mathrm{E}),(\mathrm{G}, \mathrm{E})$ are disjoint soft sets then $(\mathrm{F}, \mathrm{E}) \widetilde{\cap}(\mathrm{G}, \mathrm{E})=\widetilde{\Phi}$ Then $(\mathrm{F}, \mathrm{E})^{\prime}=(\mathrm{G}, \mathrm{E}),(\mathrm{G}, \mathrm{E})^{\prime}=(\mathrm{F}, \mathrm{E})$

Hence (F,E), (G,E) are both soft open sets in $\widetilde{Y}$.

$(\Leftarrow)$ Conversely let the disjoint soft sets $(F, E),(G, E)$ be both soft open and soft closed in

$(\mathrm{F}, \mathrm{E}) \widetilde{\sim}(\mathrm{G}, \mathrm{E})=\widetilde{\mathrm{Y}}$. To prove that $(\mathrm{F}, \mathrm{E})$ and $(\mathrm{G}, \mathrm{E})$ are soft separated in $\widetilde{\mathrm{X}}$

Since $(\mathrm{F}, \mathrm{E})$ is soft closed in $\widetilde{Y}$ then

$(\mathrm{F}, \mathrm{E})=\mathrm{cl}_{\mathrm{y}}(\mathrm{F}, \mathrm{E})=\mathrm{cl}_{\mathrm{x}}(\mathrm{F}, \mathrm{E}) \widetilde{\cap} \widetilde{\mathrm{Y}}=\mathrm{cl}_{\mathrm{x}}(\mathrm{F}, \mathrm{E}) \widetilde{\cap}[(\mathrm{F}, \mathrm{E}) \widetilde{\sim}(\mathrm{G}, \mathrm{E})]$

$=\left[\mathrm{cl}_{\mathrm{x}}(\mathrm{F}, \mathrm{E}) \widetilde{\cap}(\mathrm{F}, \mathrm{E})\right] \widetilde{\sim}\left[\mathrm{cl}_{\mathrm{x}}(\mathrm{F}, \mathrm{E}) \widetilde{\cap}(\mathrm{G}, \mathrm{E})\right]=(\mathrm{F}, \mathrm{E}) \widetilde{\sim}\left[\mathrm{cl}_{\mathrm{x}}(\mathrm{F}, \mathrm{E}) \widetilde{\cap}(\mathrm{G}, \mathrm{E})\right]$.

$\therefore(\mathrm{F}, \mathrm{E})=(\mathrm{F}, \mathrm{E}) \widetilde{\sim}\left[\mathrm{cl}_{\mathrm{x}}(\mathrm{F}, \mathrm{E}) \widetilde{\cap}(\mathrm{G}, \mathrm{E})\right]$

Then $\mathrm{cl}_{\mathrm{x}}(\mathrm{F}, \mathrm{E}) \widetilde{\cap}(\mathrm{G}, \mathrm{E})=\widetilde{\Phi}$ similarly $(\mathrm{F}, \mathrm{E}) \widetilde{\cap} \mathrm{cl}_{\mathrm{x}}(\mathrm{G}, \mathrm{E})=\widetilde{\Phi}$

Hence $(F, E)$ and $(G, E)$ are soft separated in $(X, \widetilde{T}, E)$.

Theorem 3.8. A S.T.S. $(X, \widetilde{T}, E)$ is soft disconnected iff there exist a non null soft proper subset of $(X, \widetilde{T}, E)$ which is soft clopen over $\mathrm{X}$.

Proof $(\Rightarrow)$ Let $(X, \widetilde{T}, E)$ be soft disconnected space then $\widetilde{X}$ is the union of two non-null soft open subsets $(F, E)$, $(\mathrm{G}, \mathrm{E})$ s. t. their intersections is the null set, hence $(\mathrm{F}, \mathrm{E}),(\mathrm{G}, \mathrm{E})$ are both non- null soft clopen proper subsets of $(\mathrm{X}, \widetilde{\mathrm{T}}, \mathrm{E})$.

$(\Leftarrow)$ Let $(F, E)$ be a non-null soft proper subset of $(X, \widetilde{T}, E)$ which is soft clopen over $X$ then $(F, E)$ ' is soft clopen s. t. $\widetilde{X}$ is the union of two non-null soft open subsets $(\mathrm{F}, \mathrm{E}),(\mathrm{F}, \mathrm{E})^{\prime}$ and their intersections is the null set, hence $(\mathrm{X}, \widetilde{\mathrm{T}}, \mathrm{E})$ is soft disconnected space .

Corollary 3.9. A S.T.S. (X, $\widetilde{T}, E)$ is soft connected iff the only soft subsets in $(X, \widetilde{T}, E)$ that are both soft clopen over $\mathrm{X}$ are $\widetilde{\Phi}, \widetilde{X}$.

Proof Obvious .

Theorem 3.10. A S.T.S.(X, $\widetilde{T}, E)$ is soft disconnected iff one of the following statements holds

(i) $\widetilde{X}$ is the union of two non-null soft disjoint soft open sets.

(ii) $\widetilde{\mathrm{X}}$ is the union of two non-null soft disjoint soft closed sets.

Proof: $(\Rightarrow)$ Let $\widetilde{X}$ be soft disconnected. Then there exist a non-empty proper subset $(F, E)$ of $\widetilde{X}$ which is soft clopen then (F,E)' is also both soft clopen,

$(\mathrm{F}, \mathrm{E}) \widetilde{\sim}(\mathrm{F}, \mathrm{E})^{\prime}=\widetilde{\mathrm{X}}$, Hence the sets $(\mathrm{F}, \mathrm{E})$ and $(\mathrm{F}, \mathrm{E})^{\prime}$ satisfy (i), (ii),

$(\Leftarrow)$ Let $\widetilde{\mathrm{X}}=(\mathrm{F}, \mathrm{E}) \widetilde{\sim}(\mathrm{G}, \mathrm{E})$ and $(\mathrm{F}, \mathrm{E}) \widetilde{\cap}(\mathrm{G}, \mathrm{E})=\widetilde{\Phi}$ where $(\mathrm{F}, \mathrm{E}),(\mathrm{G}, \mathrm{E})$ are non-null soft open sets, it follows that $(\mathrm{F}, \mathrm{E})=(\mathrm{G}, \mathrm{E})$ ' so that $(\mathrm{F}, \mathrm{E})$ is soft closed , since $(\mathrm{G}, \mathrm{E})$ is non-null , $(\mathrm{F}, \mathrm{E})$ is soft proper subset of $(\mathrm{X}, \widetilde{T}, \mathrm{E})$ thus $(\mathrm{F}, \mathrm{E})$ is a non-null proper soft subset of $(\mathrm{X}, \widetilde{\mathrm{T}}, \mathrm{E})$ which is soft clopen ,Hence by theorem (3.8.) (X, $, \mathrm{T}, \mathrm{E})$ is soft disconnected, (for the second case) $(\mathrm{F}, \mathrm{E}),(\mathrm{G}, \mathrm{E})$ are non-null soft closed sets, then $(\mathrm{F}, \mathrm{E})=(\mathrm{G}, \mathrm{E})$ ' so that $(\mathrm{F}, \mathrm{E})$ is soft open, since $(\mathrm{G}, \mathrm{E})$ is non-null, $(\mathrm{F}, \mathrm{E})$ is proper subset of $(\mathrm{X}, \widetilde{\mathrm{T}}, \mathrm{E})$ which is soft clopen then by theorem (3.8.) $(\mathrm{X}, \widetilde{\mathrm{T}}, \mathrm{E})$ is soft disconnected space .

Theorem 3.11. Let $(X, \widetilde{T}, E)$ be S.T.S. and let $\left(Y, \widetilde{T}_{Y}, E\right)$ be soft subspace of $(X, \widetilde{T}, E)$ then $\left(Y, \widetilde{T}_{Y}, E\right)$ is soft disconnected soft space iff there exist non-null soft sets $(\mathrm{G}, \mathrm{E}),(\mathrm{H}, \mathrm{E})$ is soft clopen in $(\mathrm{X}, \widetilde{\mathrm{T}}, \mathrm{E})$ such that

$(\mathrm{G}, \mathrm{E}) \widetilde{\cap} \widetilde{\mathrm{Y}} \neq \widetilde{\Phi},(\mathrm{H}, \mathrm{E}) \widetilde{\cap} \widetilde{\mathrm{Y}} \neq \widetilde{\Phi}$ then $(\mathrm{G}, \mathrm{E}) \widetilde{\cap}(\mathrm{H}, \mathrm{E}) \widetilde{\subseteq} \widetilde{\mathrm{X}} \backslash \widetilde{\mathrm{Y}}$ and $\widetilde{\mathrm{Y}} \widetilde{\subseteq}(\mathrm{G}, \mathrm{E}) \widetilde{\sim}(\mathrm{H}, \mathrm{E})$

Proof By (definition 3.6.) (Y,T ',E) is soft disconnected iff there exist non-null soft sets (G,E), (H,E) are soft clopen in $(X, \widetilde{T}, E)$ such that $(\mathrm{G}, \mathrm{E}) \widetilde{\cap} \widetilde{\mathrm{Y}} \neq \widetilde{\Phi},(\mathrm{H}, \mathrm{E}) \widetilde{\cap} \widetilde{\mathrm{Y}} \neq \widetilde{\Phi}$,

$[(\mathrm{G}, \mathrm{E}) \widetilde{\cap} \widetilde{\mathrm{Y}}] \widetilde{\cap}[(\mathrm{H}, \mathrm{E}) \widetilde{\cap} \widetilde{\mathrm{Y}}]=\widetilde{\Phi}$

$\Leftrightarrow[(\mathrm{G}, \mathrm{E}) \widetilde{\cap}(\mathrm{H}, \mathrm{E})] \widetilde{\cap} \widetilde{\mathrm{Y}}=\widetilde{\Phi}$

$\Leftrightarrow(\mathrm{G}, \mathrm{E}) \widetilde{\cap}(\mathrm{H}, \mathrm{E}) \widetilde{\subseteq} \widetilde{\mathrm{X}} \mid \widetilde{\mathrm{Y}}$

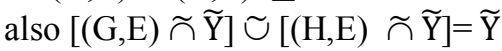

$\Leftrightarrow[(\mathrm{G}, \mathrm{E}) \widetilde{\sim}(\mathrm{H}, \mathrm{E})] \widetilde{\cap} \widetilde{\mathrm{Y}}=\widetilde{\mathrm{Y}}$

$\Leftrightarrow \widetilde{\mathrm{Y}} \widetilde{\subseteq}(\mathrm{G}, \mathrm{E}) \widetilde{\sim}(\mathrm{H}, \mathrm{E})$.

Remark 3.12. The union of soft connected spaces not necessary soft connected.

Example 3.13. let $X=\left\{h_{1}, h_{2}\right\}, E=\left\{e_{1}, e_{2}, e_{3}, e_{4}\right\}$ then

$\left.\widetilde{\mathrm{T}}_{\mathrm{M}}=\{\widetilde{\Phi}, \widetilde{\mathrm{X}},\{\mathrm{X}, \Phi, \mathrm{X}, \Phi\}, \widetilde{\Phi}\}\right\}$ and $\widetilde{\mathrm{T}}_{\mathrm{N}}=\{\widetilde{\Phi}, \widetilde{\mathrm{X}}, \widetilde{\Phi},\{\Phi, \mathrm{X}, \Phi, \mathrm{X}\}\}$ 
Are both soft connected spaces but

$\widetilde{\mathrm{T}}_{\mathrm{M}} \widetilde{\cup}_{\mathrm{N}}=\widetilde{\mathrm{T}}_{\mathrm{H}}=\{\widetilde{\Phi}, \widetilde{\mathrm{X}},\{\mathrm{X}, \Phi, \mathrm{X}, \Phi\},\{\Phi, \mathrm{X}, \Phi, \mathrm{X}\}\}$ is soft disconnected space.

Remark 3.14. The intersection of soft connected spaces not necessary soft connected.

Example 3.15. $\mathrm{X}=\left\{\mathrm{h}_{1}, \mathrm{~h}_{2}\right\}, \mathrm{E}=\left\{\mathrm{e}_{1}, \mathrm{e}_{2}, \mathrm{e}_{3}, \mathrm{e}_{4}\right\}$

$\left.\widetilde{\mathrm{T}}_{\mathrm{M}}=\left\{\widetilde{\Phi}, \widetilde{\mathrm{X}},\left\{\left\{\mathrm{h}_{1}, \mathrm{~h}_{2}\right\}\right\}, \Phi, \Phi\right\}, \widetilde{\mathrm{X}}\right\}, \widetilde{\mathrm{T}}_{\mathrm{G}}=\left\{\widetilde{\Phi},\left\{\Phi,\left\{\mathrm{h}_{1}, \mathrm{~h}_{2}\right\},\left\{\mathrm{h}_{1}, \mathrm{~h}_{2}\right\}\right\}, \widetilde{\mathrm{X}}, \widetilde{\mathrm{X}}\right\}$

Are both soft connected spaces but

$\widetilde{\mathrm{T}}_{\mathrm{M}} \widetilde{\mathrm{h}}_{\mathrm{G}}=\left\{\widetilde{\Phi},\left\{\Phi,\left\{\mathrm{h}_{1}, \mathrm{~h}_{2}\right\},\left\{\mathrm{h}_{1}, \mathrm{~h}_{2}\right\}\right\},\left\{\left\{\mathrm{h}_{1}, \mathrm{~h}_{2}\right\}, \Phi, \Phi\right\}, \widetilde{\mathrm{X}}\right\}$ is soft disconnected space.

Theorem 3.16. Let $(F, E)$ be soft subset of S.T.S. $(X, T, E)$ and $(F, E)$ is soft connected then cl(F,E) is soft connected.

Proof Suppose $\mathrm{cl}(\mathrm{F}, \mathrm{E})=(\mathrm{G}, \mathrm{E}) \widetilde{\sim}(\mathrm{C}, \mathrm{E})$ s.t. $(\mathrm{G}, \mathrm{E}) \widetilde{\cap}(\mathrm{C}, \mathrm{E})=\widetilde{\Phi}$

then $(\mathrm{F}, \mathrm{E}) \widetilde{\cap} \mathrm{cl}(\mathrm{F}, \mathrm{E})=(\mathrm{F}, \mathrm{E}) \widetilde{\cap}[(\mathrm{G}, \mathrm{E}) \widetilde{\sim}(\mathrm{C}, \mathrm{E})]$

$=[(\mathrm{F}, \mathrm{E}) \widetilde{\cap}(\mathrm{G}, \mathrm{E})] \widetilde{\sim}[(\mathrm{F}, \mathrm{E}) \widetilde{\cap}(\mathrm{C}, \mathrm{E})]$

$[(\mathrm{F}, \mathrm{E}) \widetilde{\cap}(\mathrm{G}, \mathrm{E})] \widetilde{\sim}[(\mathrm{F}, \mathrm{E}) \widetilde{\cap}(\mathrm{C}, \mathrm{E})]=(\mathrm{F}, \mathrm{E})$

$(\mathrm{F}, \mathrm{E}) \widetilde{\cap} \widetilde{\Phi}=(\mathrm{F}, \mathrm{E}) \widetilde{\cap}[(\mathrm{G}, \mathrm{E}) \widetilde{\cap}(\mathrm{C}, \mathrm{E})]$

$[(\mathrm{F}, \mathrm{E}) \widetilde{\cap}(\mathrm{G}, \mathrm{E})] \widetilde{\cap}[(\mathrm{F}, \mathrm{E}) \widetilde{\cap}(\mathrm{C}, \mathrm{E})]=\widetilde{\Phi}$

Then from (1), (2) (F,E) is soft separated $\mathrm{c} ! \therefore \mathrm{cl}(\mathrm{F}, \mathrm{E})$ must be soft connected .

\section{Soft Hereditary Property And Soft Product On Soft Connected Spaces}

In this section we will define soft hereditary property and study soft hereditary property on soft connected spaces .

Definition4.1. A property P of S.T.S. (X, $\widetilde{T}, E)$ (is soft hereditary) iff every soft subspace $\left(Y, \widetilde{T}_{Y}, E\right)$ of $(X, \widetilde{T}, E)$ also possesses a property $P$.

Example 4.2. Let $\mathrm{X}=\left\{\mathrm{h}_{1}, \mathrm{~h}_{2}, \mathrm{~h}_{3}\right\}, \mathrm{E}=\left\{\mathrm{e}_{1}, \mathrm{e}_{2}, \mathrm{e}_{3}\right\}$

$\widetilde{\mathrm{T}}_{\mathrm{x}}=\left\{\widetilde{\Phi}, \widetilde{\mathrm{X}},\left\{\left\{\mathrm{h}_{1}, \mathrm{~h}_{3}\right\}, \Phi, \Phi\right\},\left\{\left\{\mathrm{h}_{1}, \mathrm{~h}_{3}\right\},\left\{\mathrm{h}_{1} \mathrm{~h}_{3}\right\},\left\{\mathrm{h}_{1}, \mathrm{~h}_{3}\right\}\right\}\right.$

$\left(\mathrm{X}, \widetilde{\mathrm{T}}_{\mathrm{X}}, \mathrm{E}\right)$ is soft connected space, take $\mathrm{Y}=\left\{\mathrm{h}_{1}, \mathrm{~h}_{3}\right\}$

$\widetilde{\mathrm{Y}}=\left\{\left\{\mathrm{h}_{1}, \mathrm{~h}_{3}\right\},\left\{\mathrm{h}_{1} \mathrm{~h}_{3}\right\},\left\{\mathrm{h}_{1}, \mathrm{~h}_{3}\right\}\right\} \widetilde{\in} \widetilde{\mathrm{T}}_{\mathrm{x}}$ then $\widetilde{\mathrm{T}}_{\mathrm{Y}}=\left\{\widetilde{\Phi}_{\mathrm{Y}}, \widetilde{\mathrm{Y}},\left\{\left\{\mathrm{h}_{1}, \mathrm{~h}_{3}\right\}, \Phi, \Phi\right\}, \widetilde{\mathrm{Y}}\right\}$

$\therefore\left(\mathrm{Y}, \widetilde{\mathrm{T}}_{\mathrm{Y}}, \mathrm{E}\right)$ is soft connected subspace of soft connected space $\left(\mathrm{X}, \widetilde{\mathrm{T}}_{\mathrm{X}}, \mathrm{E}\right)$.

Example 4.3. Let $\mathrm{X}=\left\{\mathrm{h}_{1}, \mathrm{~h}_{2}, \mathrm{~h}_{3}\right\}, \mathrm{E}=\left\{\mathrm{e}_{1}, \mathrm{e}_{2}, \mathrm{e}_{3}\right\}$

$\widetilde{\mathrm{T}}_{\mathrm{x}}=\left\{\widetilde{\Phi}, \widetilde{\mathrm{X}},\left\{\left\{\mathrm{h}_{1}, \mathrm{~h}_{3}\right\}, \Phi, \Phi\right\},\left\{\left\{\mathrm{h}_{1}, \mathrm{~h}_{2}\right\}, \Phi, \Phi\right\},\left\{\left\{\mathrm{h}_{2}\right\}, \Phi, \Phi\right\},\left\{\left\{\mathrm{h}_{1}, \mathrm{~h}_{2}, \mathrm{~h}_{3}\right\}, \Phi, \Phi\right\}\right\}$

$\left(\mathrm{X}, \widetilde{\mathrm{T}}_{\mathrm{X}}, \mathrm{E}\right)$ is soft connected space,

take $\mathrm{Y}=\left\{\mathrm{h}_{1}, \mathrm{~h}_{2}\right\}, \widetilde{\mathrm{Y}}=\left\{\left\{\mathrm{h}_{1}, \mathrm{~h}_{2}\right\},\left\{\mathrm{h}_{1} \mathrm{~h}_{2}\right\},\left\{\mathrm{h}_{1}, \mathrm{~h}_{2}\right\}\right\} \widetilde{\notin} \widetilde{\mathrm{T}}_{\mathrm{x}}$ then

$\left.\widetilde{\mathrm{T}}_{\mathrm{Y}}=\left\{\widetilde{\Phi}_{\mathrm{Y}}, \widetilde{\mathrm{Y}},\left\{\left\{\mathrm{h}_{1}\right\}, \Phi, \Phi\right\},\left\{\left\{\mathrm{h}_{1}, \mathrm{~h}_{2}\right\}, \Phi, \Phi\right\},\left\{\left\{\mathrm{h}_{2}\right\}, \Phi, \Phi\right\}\right\},\left\{\left\{\mathrm{h}_{1}, \mathrm{~h}_{2}\right\}, \Phi, \Phi\right\}\right\}$

$\therefore\left(\mathrm{Y}, \widetilde{\mathrm{T}}_{\mathrm{Y}}, \mathrm{E}\right)$ is soft connected subspace of soft connected space $\left(\mathrm{X}, \widetilde{\mathrm{T}}_{\mathrm{X}}, \mathrm{E}\right)$.

Notice that for the soft set $\left\{\left\{\mathrm{h}_{1}\right\}, \Phi, \Phi\right\}$ it's complement in a S.T.S. (Y, $\left.\widetilde{\mathrm{T}}_{\mathrm{Y}}, \mathrm{E}\right)$

Is $\left\{\left\{\mathrm{h}_{2}\right\},\left\{\mathrm{h}_{1} \mathrm{~h}_{2}\right\},\left\{\mathrm{h}_{1}, \mathrm{~h}_{2}\right\}\right\} \widetilde{\notin} \widetilde{\mathrm{T}}_{\mathrm{Y}}$ so $\left\{\left\{\mathrm{h}_{1}\right\}, \Phi, \Phi\right\}$ is not soft clopen then by (Theorem 3.8.) (Y, $\left.\widetilde{\mathrm{T}}_{\mathrm{Y}}, \mathrm{E}\right)$ can't be soft disconnected.

Example 4.4. Let $\mathrm{X}=\left\{\mathrm{h}_{1}, \mathrm{~h}_{2}, \mathrm{~h}_{3}\right\}, \widetilde{\mathrm{T}}_{\mathrm{x}}=\left\{\widetilde{\Phi}, \widetilde{\mathrm{X}},\left\{\mathrm{h}_{1}, \Phi, \Phi\right\},\left\{\mathrm{h}_{1}, \mathrm{~h}_{2}, \Phi\right\}\right\}$

$\left(X, \widetilde{T}_{x}, E\right)$ is soft connected space let $\left.\mathrm{Y}=\left\{\mathrm{h}_{1}, \mathrm{~h}_{3}\right\}, \widetilde{\mathrm{Y}}=\left\{\mathrm{h}_{1} \mathrm{~h}_{3}\right\},\left\{\mathrm{h}_{1} \mathrm{~h}_{3}\right\},\left\{\mathrm{h}_{1} \mathrm{~h}_{3}\right\}\right\} \widetilde{\notin} \widetilde{\mathrm{T}}_{\mathrm{x}}$

$\widetilde{Y}$ is not soft open an even not soft closed subspace of $\left(X, \widetilde{T}_{x}, E\right)$

$\widetilde{\mathrm{T}}_{\mathrm{Y}}=\left\{\widetilde{\Phi}_{\mathrm{Y}}, \widetilde{\mathrm{Y}},\left\{\left\{\mathrm{h}_{1}\right\}, \Phi, \Phi\right\},\left\{\left\{\mathrm{h}_{1}\right\}, \Phi, \Phi\right\}\right\}$, Then $\left(\widetilde{\mathrm{Y}}, \widetilde{\mathrm{T}}_{\mathrm{Y}}, \mathrm{E}\right)$ is soft connected space

Example 4.5. Let $\mathrm{X}=\left\{\mathrm{h}_{1}, \mathrm{~h}_{2}, \mathrm{~h}_{3}\right\}, \mathrm{E}=\left\{\mathrm{e}_{1}, \mathrm{e}_{2}, \mathrm{e}_{3}\right\}$

$\widetilde{\mathrm{T}}_{\mathrm{X}}=\left\{\widetilde{\Phi}, \widetilde{\mathrm{X}},\left\{\left\{\mathrm{h}_{1}, \mathrm{~h}_{3}\right\}, \Phi, \Phi\right\},\{\Phi, \mathrm{X}, \mathrm{X}\},\left\{\left\{\mathrm{h}_{1}, \mathrm{~h}_{3}\right\}, \mathrm{X}, \mathrm{X}\right\}\right\}$

$\left(\mathrm{X}, \widetilde{\mathrm{T}}_{\mathrm{X}}, \mathrm{E}\right)$ is soft connected space, take $\mathrm{Y}=\left\{\mathrm{h}_{1}, \mathrm{~h}_{3}\right\}$

$\widetilde{\mathrm{Y}}=\left\{\left\{\mathrm{h}_{1}, \mathrm{~h}_{3}\right\},\left\{\mathrm{h}_{1} \mathrm{~h}_{3}\right\},\left\{\mathrm{h}_{1}, \mathrm{~h}_{3}\right\}\right\} \widetilde{\notin} \widetilde{\mathrm{T}}_{\mathrm{x}}$ then

$\widetilde{\mathrm{T}}_{\mathrm{Y}}=\left\{\widetilde{\Phi}_{\mathrm{Y}}, \widetilde{\mathrm{Y}},\left\{\left\{\mathrm{h}_{1}, \mathrm{~h}_{3}\right\}, \Phi, \Phi\right\},\left\{\Phi,\left\{\mathrm{h}_{1}, \mathrm{~h}_{3}\right\},\left\{\mathrm{h}_{1}, \mathrm{~h}_{3}\right\}\right\}, \widetilde{\mathrm{Y}}\right\}$

Then $\left(\mathrm{Y}, \widetilde{\mathrm{T}}_{\mathrm{Y}}, \mathrm{E}\right)$ is soft disconnected subspace of soft connected space.

Example 4.6. Let $\mathrm{X}=\left\{\mathrm{h}_{1}, \mathrm{~h}_{2}, \mathrm{~h}_{3}\right\}, \mathrm{E}=\left\{\mathrm{e}_{1}, \mathrm{e}_{2}, \mathrm{e}_{3}\right\}$

$\widetilde{\mathrm{T}}_{\mathrm{x}}=\left\{\widetilde{\Phi}, \widetilde{\mathrm{X}},\left\{\left\{\mathrm{h}_{1}\right\},\left\{\mathrm{h}_{1}\right\},\left\{\mathrm{h}_{1}\right\}\right\},\left\{\left\{\mathrm{h}_{2}\right\},\left\{\mathrm{h}_{2}\right\},\left\{\mathrm{h}_{2}\right\}\right\},\left\{\left\{\mathrm{h}_{1}, \mathrm{~h}_{2}\right\},\left\{\mathrm{h}_{1} \mathrm{~h}_{2}\right\},\left\{\mathrm{h}_{1} \mathrm{~h}_{2}\right\}\right\}\right.$

$\left(\mathrm{X}, \widetilde{\mathrm{T}}_{\mathrm{x}}, \mathrm{E}\right)$ is soft connected space, take $\mathrm{Y}=\left\{\mathrm{h}_{1}, \mathrm{~h}_{2}\right\}, \widetilde{\mathrm{Y}}=\left\{\left\{\mathrm{h}_{1}, \mathrm{~h}_{2}\right\},\left\{\mathrm{h}_{1} \mathrm{~h}_{2}\right\},\left\{\mathrm{h}_{1}, \mathrm{~h}_{2}\right\}\right\} \widetilde{\in} \widetilde{\mathrm{T}}_{\mathrm{x}}$

Then $\widetilde{\mathrm{T}}_{\mathrm{Y}}=\left\{\widetilde{\Phi}_{\mathrm{Y}}, \widetilde{\mathrm{Y}},\left\{\left\{\mathrm{h}_{1}\right\},\left\{\mathrm{h}_{1}\right\},\left\{\mathrm{h}_{1}\right\}\right\},\left\{\left\{\mathrm{h}_{2}\right\},\left\{\mathrm{h}_{2}\right\},\left\{\mathrm{h}_{2}\right\}\right\}, \widetilde{\mathrm{Y}}\right\}$

Then $\left(\mathrm{Y}, \widetilde{\mathrm{T}}_{\mathrm{Y}}, \mathrm{E}\right)$ is soft disconnected subspace of soft connected space

Remark 4.7. From the previous examples we conclude that the soft connectedness is not necessary a soft hereditary property even on soft open set which is different from general topology .

Definition 4.8. The soft connected topological space in which every non null soft proper set is soft connected is called completely soft connected space.

Remark 4.9. Completely soft connected space is soft connected space but the converse is not necessary true. 
Example 4.10. From example (4.3.) $\left(\mathrm{Y}, \widetilde{\mathrm{T}}_{\mathrm{Y}}, \mathrm{E}\right)$ is soft connected space but not completely soft connected space since the soft open set $\left\{\left\{\mathrm{h}_{1}, \mathrm{~h}_{2}\right\}, \Phi, \Phi\right\}$ in $\widetilde{\mathrm{T}}_{\mathrm{Y}}$ is soft disconnected .

\section{Soft Product Of Soft Connected And Soft Disconnected Space}

In this section the soft sets $(F, E),(G, E)$ have the same set of parameters $E$.

Definition5.1.[1] Let $\left(\mathrm{F}, \mathrm{E}_{1}\right)$ and $\left(\mathrm{G}, \mathrm{E}_{2}\right)$ be soft sets in $\mathrm{SS}(\mathrm{X})_{\mathrm{E} 1}$, and $\mathrm{SS}(\mathrm{Y})_{\mathrm{E} 2}$ respectively. Then the Cartesian product of $\left(\mathrm{F}, \mathrm{E}_{1}\right)$ and $\left(\mathrm{G}, \mathrm{E}_{2}\right)$ denoted by $\left(\mathrm{F} \times \mathrm{G}, \mathrm{E}_{1} \times \mathrm{E}_{2}\right)$ in $\mathrm{SS}(\mathrm{X} \times \mathrm{Y})_{\mathrm{E} 1 \times \mathrm{E} 2}$ is defined as :

$(\mathrm{F} \times \mathrm{G})\left(\mathrm{e}_{1}, \mathrm{e}_{2}\right)=\mathrm{F}\left(\mathrm{e}_{1}\right) \times \mathrm{G}\left(\mathrm{e}_{2}\right)$

Definition 5.2.[1] Let $\left(X, \widetilde{T}_{1}, \mathrm{E}_{1}\right)$ and $\left(\mathrm{Y}, \widetilde{\mathrm{T}}_{2}, \mathrm{E}_{2}\right)$ be soft spaces

let $\mathrm{B}=\left\{\left(\mathrm{F}, \mathrm{E}_{1}\right) \times\left(\mathrm{G}, \mathrm{E}_{2}\right) \mid\left(\mathrm{F}, \mathrm{E}_{1}\right) \in \widetilde{\mathrm{T}}_{1},\left(\mathrm{G}, \mathrm{E}_{2}\right) \in \widetilde{\mathrm{T}}_{2}\right\}$ and $\widetilde{\mathrm{T}}$ be the collection of all arbitrary union of elements of $\mathrm{B}$, Then $\widetilde{T}$ is a (soft topology over $\mathrm{X} \times \mathrm{Y}$ )

and $\left(\mathrm{X} \times \mathrm{Y}, \widetilde{\mathrm{T}}, \mathrm{E}_{1} \times \mathrm{E}_{2}\right)$ is called (soft product topological space over $\mathrm{X} \times \mathrm{Y}$ )

Proposition 5.3.[9] $\Phi_{\mathrm{E} 1} \times\left(\mathrm{F}_{2}, \mathrm{E}_{2}\right)=\left(\mathrm{F}_{1}, \mathrm{E}_{1}\right) \times \Phi_{\mathrm{E} 2}=\Phi_{\mathrm{E} 1 \times \mathrm{E} 2}$

Theorem 5.4.[9] The soft product of two soft connected spaces is soft connected space .

Example 5.5. Let $X=\{h\}, E=\left\{\mathrm{e}_{1}, \mathrm{e}_{2}\right\},\left(\mathrm{F}_{1}\right)\left(\mathrm{e}_{1}\right)=\left(\mathrm{F}_{1}\right)\left(\mathrm{e}_{2}\right)=\Phi$

$\left(\mathrm{F}_{2}\right)\left(\mathrm{e}_{1}\right)=\left(\mathrm{F}_{2}\right)\left(\mathrm{e}_{2}\right)=\mathrm{X}$, then $\widetilde{T}_{1}$ is soft connected space and

$(\mathrm{F}, \widetilde{\mathrm{T}}, \mathrm{E}) \times(\mathrm{F}, \widetilde{\mathrm{T}} \mathrm{i}, \mathrm{E})=(\mathrm{F} \times \mathrm{F}, \widetilde{\mathrm{T}}, \mathrm{E} \times \mathrm{E}),\left(\mathrm{F}_{\mathrm{i}} \times \mathrm{F}_{\mathrm{i}}\right)\left(\mathrm{e}_{\mathrm{i}}, \mathrm{e}_{\mathrm{i}}\right)=\mathrm{F}_{\mathrm{i}}\left(\mathrm{e}_{\mathrm{i}}\right) \times \mathrm{F}_{\mathrm{i}}\left(\mathrm{e}_{\mathrm{i}}\right), \mathrm{i}=1,2$

$\left(\mathrm{F}_{1} \times \mathrm{F}_{1}, \mathrm{E} \times \mathrm{E}\right)=\left\{\left(\mathrm{F}_{1} \times \mathrm{F}_{1}\right)\left(\mathrm{e}_{1}, \mathrm{e}_{1}\right),\left(\mathrm{F}_{1} \times \mathrm{F}_{1}\right)\left(\mathrm{e}_{1}, \mathrm{e}_{2}\right),\left(\mathrm{F}_{1} \times \mathrm{F}_{1}\right)\left(\mathrm{e}_{2}, \mathrm{e}_{1}\right),\left(\mathrm{F}_{1} \times \mathrm{F}_{1}\right)\left(\mathrm{e}_{2}, \mathrm{e}_{2}\right)\right\}=\widetilde{\Phi}_{\mathrm{E} \times \mathrm{E}}$

$\left(\mathrm{F}_{1} \times \mathrm{F}_{2}, \mathrm{E} \times \mathrm{E}\right)=\left\{\left(\mathrm{F}_{1} \times \mathrm{F}_{2}\right)\left(\mathrm{e}_{1}, \mathrm{e}_{1}\right),\left(\mathrm{F}_{1} \times \mathrm{F}_{2}\right)\left(\mathrm{e}_{1}, \mathrm{e}_{2}\right),\left(\mathrm{F}_{1} \times \mathrm{F}_{2}\right)\left(\mathrm{e}_{2}, \mathrm{e}_{1}\right),\left(\mathrm{F}_{1} \times \mathrm{F}_{2}\right)\left(\mathrm{e}_{2}, \mathrm{e}_{2}\right)\right\}=\widetilde{\Phi}_{\mathrm{E} \times \mathrm{E}}$

$\left(\mathrm{F}_{2} \times \mathrm{F}_{1}, \mathrm{E} \times \mathrm{E}\right)=\left\{\left(\mathrm{F}_{2} \times \mathrm{F}_{1}\right)\left(\mathrm{e}_{1}, \mathrm{e}_{1}\right),\left(\mathrm{F}_{2} \times \mathrm{F}_{1}\right)\left(\mathrm{e}_{1}, \mathrm{e}_{2}\right),\left(\mathrm{F}_{2} \times \mathrm{F}_{1}\right)\left(\mathrm{e}_{2}, \mathrm{e}_{1}\right),\left(\mathrm{F}_{2} \times \mathrm{F}_{1}\right)\left(\mathrm{e}_{2}, \mathrm{e}_{2}\right)\right\}=\widetilde{\Phi}_{\mathrm{E} \times \mathrm{E}}$

$\left(\mathrm{F}_{2} \times \mathrm{F}_{2}, \mathrm{E} \times \mathrm{E}\right)=\left\{\left(\mathrm{F}_{2} \times \mathrm{F}_{2}\right)\left(\mathrm{e}_{2}, \mathrm{e}_{2}\right),\left(\mathrm{F}_{2} \times \mathrm{F}_{2}\right)\left(\mathrm{e}_{2}, \mathrm{e}_{2}\right),\left(\mathrm{F}_{2} \times \mathrm{F}_{2}\right)\left(\mathrm{e}_{2}, \mathrm{e}_{2}\right),\left(\mathrm{F}_{2} \times \mathrm{F}_{2}\right)\left(\mathrm{e}_{2}, \mathrm{e}_{2}\right)\right\}=\widetilde{\mathrm{X}} \times \widetilde{\mathrm{X}}$

$\left(\mathrm{F}_{1} \times \mathrm{F}_{1}, \mathrm{E} \times \mathrm{E}\right),\left(\mathrm{F}_{1} \times \mathrm{F}_{2}, \mathrm{E} \times \mathrm{E}\right),\left(\mathrm{F}_{2} \times \mathrm{F}_{1}, \mathrm{E} \times \mathrm{E}\right),\left(\mathrm{F}_{2} \times \mathrm{F}_{2}, \mathrm{E} \times \mathrm{E}\right)$ are product soft sets generate the soft product topology $\widetilde{\mathrm{T}}$, $\widetilde{\mathrm{T}}=\left\{\widetilde{\Phi}_{\mathrm{E} \times \mathrm{E}}, \widetilde{\Phi}_{\mathrm{E} \times \mathrm{E}}, \widetilde{\mathrm{X}} \times \widetilde{\mathrm{X}}\right\}$

Then it is clearly that $(\mathrm{F} \times \mathrm{F}, \widetilde{\mathrm{T}}, \mathrm{E} \times \mathrm{E})$ is soft connected product space.

Remark 5.6.The product of soft connected topological spaces with soft disconnected topological space is soft disconnected space

Example 5.7. Let $\mathrm{X} \neq \Phi, \mathrm{E}=\left\{\mathrm{e}_{1}, \mathrm{e}_{2}\right\}, \mathrm{F}_{1}\left(\mathrm{e}_{1}\right)=\mathrm{F}_{1}\left(\mathrm{e}_{2}\right)=\mathrm{X}, \mathrm{G}_{2}\left(\mathrm{e}_{1}\right)=\mathrm{G}_{2}\left(\mathrm{e}_{2}\right)=\Phi$

$\widetilde{T} \mathrm{X}=\{\widetilde{\Phi}, \widetilde{X}\}$, then $\left(X, \widetilde{T}_{X}, E\right)$ is soft connected topological space

Example 5.8. Let $\mathrm{Y}=\{\mathrm{h}\}, \mathrm{E}=\left\{\mathrm{e}_{1}, \mathrm{e}_{2}\right\}$

$\mathrm{G}_{1}\left(\mathrm{e}_{1}\right)=\mathrm{G}_{2}\left(\mathrm{e}_{2}\right)=\mathrm{G}_{3}\left(\mathrm{e}_{1}\right)=\mathrm{G}_{3}\left(\mathrm{e}_{2}\right)=\mathrm{Y}, \mathrm{G}_{1}\left(\mathrm{e}_{2}\right)=\mathrm{G}_{2}\left(\mathrm{e}_{1}\right)=\mathrm{G}_{4}\left(\mathrm{e}_{1}\right)=\mathrm{G}_{4}\left(\mathrm{e}_{2}\right)=\Phi$

$\widetilde{\mathrm{T}}_{\mathrm{Y}}=\{(\mathrm{Y}, \Phi),(\Phi, \mathrm{Y}), \widetilde{\mathrm{Y}}, \widetilde{\Phi}\}$ Then $\left(\mathrm{Y}, \widetilde{\mathrm{T}}_{\mathrm{Y}}, \mathrm{E}\right)$ is soft disconnected topological space

Example 5.9. From the previous two Examples (5.7.), (5.8.)

Let $\left(\mathrm{X}, \widetilde{\mathrm{T}}_{\mathrm{X}}, \mathrm{E}\right),\left(\mathrm{X}, \widetilde{T}_{\mathrm{Y}}, \mathrm{E}\right)$ be two soft topological spaces $\left(\mathrm{X}, \widetilde{\mathrm{T}}_{\mathrm{X}}, \mathrm{E}\right)$ soft connected

$\left(\mathrm{Y}, \widetilde{\mathrm{T}}_{\mathrm{Y}}, \mathrm{E}\right)$ soft disconnected Then $(\mathrm{X} \times \mathrm{Y}, \widetilde{\mathrm{T}}, \mathrm{E} \times \mathrm{E})$ is soft disconnected space, $\mathrm{E}=\left\{\mathrm{e}_{1}, \mathrm{e}_{2}\right\}$

$\left(\mathrm{F}_{1}, \mathrm{G}_{1}\right)\left(\mathrm{e}_{1}, \mathrm{e}_{1}\right)=\left(\mathrm{F}_{1} \times \mathrm{G}_{1}\right)\left(\mathrm{e}_{2}, \mathrm{e}_{1}\right)=\left(\mathrm{F}_{1}, \mathrm{G}_{2}\right)\left(\mathrm{e}_{1}, \mathrm{e}_{2}\right)=\left(\mathrm{F}_{1} \times \mathrm{G}_{2}\right)\left(\mathrm{e}_{2}, \mathrm{e}_{2}\right)=\mathrm{X} \times \mathrm{Y}$

And $\left(\mathrm{F}_{1} \times \mathrm{G}_{1}\right)\left(\mathrm{e}_{1}, \mathrm{e}_{2}\right)=\left(\mathrm{F}_{1} \times \mathrm{G}_{1}\right)\left(\mathrm{e}_{2}, \mathrm{e}_{2}\right)=\left(\mathrm{F}_{1} \times \mathrm{G}_{2}\right)\left(\mathrm{e}_{1}, \mathrm{e}_{1}\right)=\left(\mathrm{F}_{1} \times \mathrm{G}_{2}\right)\left(\mathrm{e}_{2}, \mathrm{e}_{1}\right)=\Phi$

Hence $\left(\mathrm{F}_{1} \times \mathrm{G}_{1}, \mathrm{E} \times \mathrm{E}\right)=\{\mathrm{X} \times \mathrm{Y}, \Phi, \mathrm{X} \times \mathrm{Y}, \Phi\},\left(\mathrm{F}_{1} \times \mathrm{G}_{2}, \mathrm{E} \times \mathrm{E}\right)=\{\Phi, \mathrm{X} \times \mathrm{Y}, \Phi, \mathrm{X} \times \mathrm{Y}\}$

The soft open sets $\left(F_{1} \times G_{1}, E \times E\right)$ and $\left(F_{1} \times G_{2}, E \times E\right)$ form a soft separation of the soft product space

$(\mathrm{X} \times \mathrm{Y}, \widetilde{\mathrm{T}}, \mathrm{E} \times \mathrm{E})$.

$\left(\mathrm{F}_{1} \times \mathrm{G}_{1}\right)\left(\mathrm{e}_{1}, \mathrm{e}_{1}\right) \cup\left(\mathrm{F}_{1} \times \mathrm{G}_{2}\right)\left(\mathrm{e}_{1}, \mathrm{e}_{1}\right)=\mathrm{X} \times \mathrm{Y} \cup \Phi=\mathrm{X} \times \mathrm{Y}$

$\left(\mathrm{F}_{1} \times \mathrm{G}_{1}\right)\left(\mathrm{e}_{1}, \mathrm{e}_{1}\right) \cap\left(\mathrm{F}_{1} \times \mathrm{G}_{2}\right)\left(\mathrm{e}_{1}, \mathrm{e}_{1}\right)=\mathrm{X} \times \mathrm{Y} \cap \Phi=\Phi$

$\left(\mathrm{F}_{1} \times \mathrm{G}_{1}\right)\left(\mathrm{e}_{1}, \mathrm{e}_{2}\right) \cup\left(\mathrm{F}_{1} \times \mathrm{G}_{2}\right)\left(\mathrm{e}_{1}, \mathrm{e}_{2}\right)=\Phi \cup \mathrm{X} \times \mathrm{Y}=\mathrm{X} \times \mathrm{Y}$

$\left(\mathrm{F}_{1} \times \mathrm{G}_{1}\right)\left(\mathrm{e}_{1}, \mathrm{e}_{2}\right) \cap\left(\mathrm{F}_{1} \times \mathrm{G}_{2}\right)\left(\mathrm{e}_{1}, \mathrm{e}_{2}\right)=\Phi$

$\left(\mathrm{F}_{1} \times \mathrm{G}_{1}\right)\left(\mathrm{e}_{2}, \mathrm{e}_{1}\right) \cup\left(\mathrm{F}_{1} \times \mathrm{G}_{2}\right)\left(\mathrm{e}_{2}, \mathrm{e}_{1}\right)=\mathrm{X} \times \mathrm{Y} \cup \Phi=\mathrm{X} \times \mathrm{Y}$

$\left(\mathrm{F}_{1} \times \mathrm{G}_{1}\right)\left(\mathrm{e}_{2}, \mathrm{e}_{1}\right) \cap\left(\mathrm{F}_{1} \times \mathrm{G}_{2}\right)\left(\mathrm{e}_{2}, \mathrm{e}_{1}\right)=\Phi$

$\left(\mathrm{F}_{1} \times \mathrm{G}_{1}\right)\left(\mathrm{e}_{2}, \mathrm{e}_{2}\right) \cup\left(\mathrm{F}_{1} \times \mathrm{G}_{2}\right)\left(\mathrm{e}_{2}, \mathrm{e}_{2}\right)=\Phi \cup \mathrm{X} \times \mathrm{Y}=\mathrm{X} \times \mathrm{Y}$

$\left(\mathrm{F}_{1} \times \mathrm{G}_{1}\right)\left(\mathrm{e}_{2}, \mathrm{e}_{2}\right) \cap\left(\mathrm{F}_{1} \times \mathrm{G}_{2}\right)\left(\mathrm{e}_{2}, \mathrm{e}_{2}\right)=\Phi$

So $\left(\mathrm{F}_{1} \times \mathrm{G}_{1}, \mathrm{E} \times \mathrm{E}\right) \widetilde{\sim}\left(\mathrm{F}_{1} \times \mathrm{G}_{2}, \mathrm{E} \times \mathrm{E}\right)=\{\mathrm{X} \times \mathrm{Y}, \mathrm{X} \times \mathrm{Y}, \mathrm{X} \times \mathrm{Y}, \mathrm{X} \times \mathrm{Y}\}=\widetilde{\mathrm{X}} \times \widetilde{\mathrm{Y}}$

And $\left(\mathrm{F}_{1} \times \mathrm{G}_{1}, \mathrm{E} \times \mathrm{E}\right) \widetilde{\cap}\left(\mathrm{F}_{1} \times \mathrm{G}_{2}, \mathrm{E} \times \mathrm{E}\right)=\{\Phi, \Phi, \Phi, \Phi\}=\widetilde{\Phi}_{\mathrm{E} \times \mathrm{E}}$

Remark 5.10. The product of soft disconnected soft topological space with soft disconnected topological space is soft disconnected topological space .

Example 5.11. From Example ( 5.8.)

$\left(\mathrm{Y}, \widetilde{\mathrm{T}}_{\mathrm{Y}}, \mathrm{E}\right) \times\left(\mathrm{Y}, \widetilde{\mathrm{T}}_{\mathrm{Y}}, \mathrm{E}\right)=\left(\mathrm{Y} \times \mathrm{Y}, \widetilde{\mathrm{T}}_{\mathrm{Y}} \times \widetilde{\mathrm{T}}_{\mathrm{Y}}, \mathrm{E} \times \mathrm{E}\right)$

$\left(\mathrm{G}_{3} \times \mathrm{G}_{1}\right)\left(\mathrm{e}_{1}, \mathrm{e}_{1}\right)=\mathrm{Y} \times \mathrm{Y}$

$\left(\mathrm{G}_{3} \times \mathrm{G}_{1}\right)\left(\mathrm{e}_{1}, \mathrm{e}_{2}\right)=\mathrm{Y} \times \Phi=\Phi$

$\left(\mathrm{G}_{3} \times \mathrm{G}_{1}\right)\left(\mathrm{e}_{2}, \mathrm{e}_{1}\right)=\mathrm{Y} \times \mathrm{Y}$

$\left(\mathrm{G}_{3} \times \mathrm{G}_{1}\right)\left(\mathrm{e}_{2}, \mathrm{e}_{2}\right)=\mathrm{Y} \times \Phi=\Phi$ 
Hence $\left(\mathrm{G}_{3} \times \mathrm{G}_{1}, \mathrm{E} \times \mathrm{E}\right)=\{\mathrm{Y} \times \mathrm{Y}, \Phi, \mathrm{Y} \times \mathrm{Y}, \Phi\}$

$\left(\mathrm{G}_{3} \times \mathrm{G}_{2}\right)\left(\mathrm{e}_{1}, \mathrm{e}_{1}\right)=\mathrm{Y} \times \Phi=\Phi$

$\left(\mathrm{G}_{3} \times \mathrm{G}_{2}\right)\left(\mathrm{e}_{1}, \mathrm{e}_{2}\right)=\mathrm{Y} \times \mathrm{Y}$

$\left(\mathrm{G}_{3} \times \mathrm{G}_{2}\right)\left(\mathrm{e}_{2}, \mathrm{e}_{1}\right)=\mathrm{Y} \times \Phi=\Phi$

$\left(\mathrm{G}_{3} \times \mathrm{G}_{2}\right)\left(\mathrm{e}_{2}, \mathrm{e}_{2}\right)=\mathrm{Y} \times \mathrm{Y}=\Phi$

Hence $\left(\mathrm{G}_{3} \times \mathrm{G}_{2}, \mathrm{E} \times \mathrm{E}\right)=\{\Phi, \mathrm{Y} \times \mathrm{Y}, \Phi, \mathrm{Y} \times \mathrm{Y}\}$

So $\left(\mathrm{G}_{1} \times \mathrm{G}_{1}, \mathrm{E} \times \mathrm{E}\right) \widetilde{\sim}\left(\mathrm{G}_{1} \times \mathrm{G}_{2}, \mathrm{E} \times \mathrm{E}\right)=\{\mathrm{Y} \times \mathrm{Y}, \mathrm{Y} \times \mathrm{Y}, \mathrm{Y} \times \mathrm{Y}, \mathrm{Y} \times \mathrm{Y}\}=\widetilde{\mathrm{Y}} \times \widetilde{\mathrm{Y}}$

And $\left(\mathrm{F}_{1} \times \mathrm{G}_{1}, \mathrm{E} \times \mathrm{E}\right) \widetilde{\cap}\left(\mathrm{F}_{1} \times \mathrm{G}_{2}, \mathrm{E} \times \mathrm{E}\right)=\{\Phi, \Phi, \Phi, \Phi\}=\widetilde{\Phi}_{\mathrm{E} \times \mathrm{E}}$

$\left(\mathrm{G}_{3} \times \mathrm{G}_{1}\right)\left(\mathrm{e}_{1}, \mathrm{e}_{1}\right)$ and $\left(\mathrm{G}_{3} \times \mathrm{G}_{2}\right)\left(\mathrm{e}_{1}, \mathrm{e}_{2}\right)$ are separation of $\widetilde{\mathrm{Y}} \times \widetilde{\mathrm{Y}}$ Therefore it is soft disconnected space.

Now we will introduce soft locally connected space on a soft element .

Definition 5.12 A S.T.S. (X, $\widetilde{T}, E$ ) is said to be (soft locally connected at a soft element $\tilde{\mathrm{X}} \widetilde{\in} \widetilde{X}$ ) if for every soft open set $(\mathrm{F}, \mathrm{E})$ containing $\tilde{\mathrm{X}}$ there is a soft connected soft open set $(\mathrm{G}, \mathrm{E})$ containing $\tilde{\mathrm{x}}$, contained in $(\mathrm{F}, \mathrm{E})$.

Remark 5.13. Every soft connected space is soft locally connected but the converse is not necessary true .

Examples 5.14.

(1) The soft indiscrete topological space is soft locally connected and soft connected.

(2) The soft discrete topological space is soft locally connected but not soft connected .

Proposition 5.15. A soft topological space $(X, \widetilde{T}, E)$ is soft locally connected iff it possesses a soft base $\beta$ consisting of a soft connected sets.

Proof $(\Rightarrow)$ Let $(X, \widetilde{T}, E)$ soft locally connected then $\forall \tilde{x} \widetilde{\in}(F, E)$, (F,E) soft open set $\exists(\mathrm{G}, \mathrm{E})$ soft open soft connected set , $\widetilde{\mathrm{x}} \widetilde{\in}(\mathrm{G}, \mathrm{E}) \widetilde{\subseteq}(\mathrm{F}, \mathrm{E})$ since $(\mathrm{F}, \mathrm{E})$ is soft open set, $(\beta, \widetilde{\mathrm{T}}, \mathrm{E})$ be a soft base for $(\mathrm{X}, \widetilde{\mathrm{T}}, \mathrm{E})$ then $(\mathrm{F}, \mathrm{E})$ $=\{\widetilde{\cup}(\mathrm{B}, \mathrm{E}):(\mathrm{B}, \mathrm{E}) \widetilde{\in} \beta, \widetilde{\mathrm{T}}, \mathrm{E})$ then $\tilde{\mathrm{x}} \widetilde{\in}(\mathrm{G}, \mathrm{E}) \widetilde{\subseteq} \widetilde{\cup}(\mathrm{B}, \mathrm{E})$

Let $\left(B_{k}, E\right)=\sup (B, E)$ then $\tilde{\mathrm{x}} \widetilde{\in}(\mathrm{G}, \mathrm{E}) \widetilde{\subseteq}\left(\mathrm{B}_{\mathrm{k}}, \mathrm{E}\right)$

$(\Leftarrow)$ Let $\tilde{x} \widetilde{\in}(G, E) \widetilde{\subseteq}\left(B_{k}, E\right),(G, E)$ soft connected soft open set, $\left(B_{k}, E\right)$ soft base then $\left(B_{k}, E\right)$ is soft open set .

Theorem 5.16. Let $(X, \widetilde{T}, E)$ and $\left(Y, \widetilde{T}^{\prime}, E\right)$ be soft locally connected spaces then

$(\mathrm{X}, \widetilde{\mathrm{T}}, \mathrm{E}) \times\left(\mathrm{Y}, \widetilde{\mathrm{T}}^{\prime}, \mathrm{E}\right)$ is soft locally connected space .

Proof Since $(X, \widetilde{T}, E)$ is soft locally connected then $(X, \widetilde{T}, E)$ possesses a soft base $\beta$ consisting of soft connected sets.

Similarly $(\mathrm{Y}, \widetilde{\mathrm{T}}, \mathrm{E})$ is soft locally connected then $(\mathrm{Y}, \widetilde{\mathrm{T}}, \mathrm{E})$ possesses a soft base $\beta^{\prime}$ consisting of soft connected sets Then $\beta^{\prime \prime}=\left\{(\mathrm{F}, \mathrm{E}) \times(\mathrm{G}, \mathrm{E}):(\mathrm{F}, \mathrm{E}) \widetilde{\in} \beta,(\mathrm{G}, \mathrm{E}) \in \beta^{\prime}\right\}$ is a soft base for the soft product space

$(X, \widetilde{T}, E) \times\left(Y, \widetilde{T}{ }^{\prime}, E\right),(F, E) \times(G, E)$ is soft connected since $(F, E),(G, E)$ are soft connected

Therefore $(\mathrm{X}, \widetilde{T}, \mathrm{E}) \times\left(\mathrm{Y}, \widetilde{\mathrm{T}}^{\prime}, \mathrm{E}\right)$ has a base consisting of soft connected sets

Therefore $(\mathrm{X}, \widetilde{\mathrm{T}}, \mathrm{E}) \times\left(\mathrm{Y}, \widetilde{\mathrm{T}}^{\prime}, \mathrm{E}\right)$ is soft locally connected.

\section{Conclusions}

In this paper we sow that In a soft topological space any two soft separated sets are soft disjoint sets but two disjoint soft sets are not necessary soft separated sets, two soft close (soft open) subsets (F,E), (G,E) of a S.T.S. (X, T,E) are soft separated if and only if they are soft disjoint,the soft intersection (soft union) of soft connected spaces not necessary soft connected, we conclude that the soft connectedness is not necessary a soft hereditary property even on soft open set which is different from general topology, by examples we show that the product of two soft disconnected spaces is soft disconnected and the product of soft connected with soft disconnected is soft disconnected which is similar as in general topology .

\section{References}

[1] A.Aygunoglu and H.Aygun, "Some notes on soft topological paces" ,Neural Comput and Applic, (2011).

[2] D. Molodtsov, "Soft Set Theory-First Results", Computers and Mathematics with Applications, Vol. 37, p.p 19-31,(1999).

[3] Dariusz Wardowski,"On a Soft Mapping and its Fixed Points",fixed point theory and application springer open journal , Vol. 182 , p.p.1-11,(2013).

[4] E.Peyghan, B.Samadi , A.Tayebi, " About soft topological spaces ", journal of new results in science, No.2, p.p 60-75(2013).

[5] E.Peyghan, B.Samadi, A.Tayebi,"on soft connectedness ",arXiv:1202.1668v1 [math.GN]8Feb(2012).

[6] Gnanambal Hango,Mrudula Ravindran ,"On soft pre open sets in soft topological spaces", international journal of mathematics research, Vol.5,No.4,p.p.399-409,(2013).

[7] J.Mahanta , P.K.Das,"On soft topological space via semi open and semi closed soft sets",NERIST,Nirjuli,Arunachal,Pradesh,791 109 , INDIA ,(2012).

[8] M. Shabir and M. Naz, "On soft topological spaces", Computers and Mathematics with Applications, 61, 17861799,(2011).

[9] P.K. Maji, R. Biswas \& A.R. Roy, "Soft Set Theory", Computer and Mathematics with Application, Vol. 45, p.p. 555-562, (2003).

[10] Sujoy Das ,S.K.Samanta,"Soft Metric", Ann. Fuzzy math. Inform., (2012). 